\title{
Antiangiogênicos no glaucoma
}

\author{
Antiangiogenics in glaucoma
}

Diogo Lucena', Riuitiro Yamane ${ }^{2}$

\section{ResUMO}

A angiogênese é um processo complexo que resulta na formação de novos vasos sangüíneos. Ocorre tanto em situações fisiológicas quanto patológicas e está envolvida com várias patologias oculares. Dentre os mediadores químicos da angiogênese, o fator de crescimento do endotélio vascular (VEGF) é reconhecido como um dos mais importantes. Nos últimos anos, agentes antiVEGF foram introduzidos para o tratamento de neovascularizações do segmento posterior com resultados muito positivos. Alguns autores demonstraram benefícios do uso dessas substâncias no controle do glaucoma em situações específicas.

Descritores: Inibidores da angiogênese/uso terapêutico; Neovascularização patológica/quimioterapia; Glaucoma neovascular/quimioterapia; Fatores de crescimento do endotélio vascular; Revisão

\footnotetext{
Médicoassistente do setor de Glaucoma do Hospital da Lagoa - Rio de Janeiro (RJ), Brasil;

2Professor Titular da Universidade Estadual do Rio de Janeiro - UERJ - Rio de Janeiro (RJ), Brasil.
} 


\section{INTRODUÇÃO}

H á mais de trinta anos, Folkman propôs o conceito de controle do leito vascular - estimulação ou inibição do crescimento de novos vasos como uma alternativa terapêutica para diversas condições clínicas ${ }^{(1)}$. Em pouco tempo, o controle da angiogênese passou a ser considerado como um dos pontos estratégicos na luta contra o câncer e inúmeras pesquisas voltaram-se nesta direção. Essas pesquisas desvendaram os principais agentes desse processo e o papel de cada um deles. Graças ao empenho desses pesquisadores, nós hoje temos meios de modular a formação de novos vasos.

Em humanos, a angiogênese ocorre tanto em situações fisiológicas, como a embriogênese e o processo de reparação tecidual, quanto patológicas ${ }^{(2-3)}$. O crescimento tumoral e metastático, a degeneração macular relacionada à idade, a artrite reumatóide e a retinopatia diabética são exemplos de doenças em que a angiogênese é parte fundamental do mecanismo fisiopatológico ${ }^{(1-3)}$.

\section{Angiogênese}

Angiogênese é o processo de obtenção de novos vasos a partir de vasos já existentes. Apesar da aparente simplicidade, trata-se de processo complexo controlado delicadamente por uma variedade de fatores promotores e inibidores da angiogênese. O papel e o mecanismo exato de ação de alguns destes fatores permanece desconhecido, ou apenas parcialmente esclarecido.

Acredita-se que o estímulo inicial básico para a angiogênese seria uma deficiência no suprimento sanguíneo e redução do aporte de oxigênio às células, causando hipóxia tecidual ${ }^{(4)}$. A hipóxia é o sinal que deflagra mecanismos intracelulares para aumentar a expressão de fatores pró-angiogênicos, como o fator de crescimento derivado do endotélio (VEGF) e o fator de crescimento dos fibroblastos (FGF). Ao mesmo tempo, a produção de fatores antiangiogênicos, como a angiostatina, é inibida. Quando os fatores pró-angiogênicos superam os fatores antiangiogênicos, as células endoteliais trocam seu estado quiescente por um estado ativo, o que é conhecido como "troca angiogênica" ${ }^{(5)}$.Tudo indica que o VEGF atua como principal fator pró-angiogênico, desempenhando um papel central neste processo.

\section{Fator de crescimento derivado do endotélio (VEGF)}

Mais do que uma única substância, o VEGF representa uma família de proteínas (Figura 1). Existem cinco tipos conhecidos: VEGF-A, VEGF-B, VEGF-C,
VEGF-D,VEGF-E ${ }^{(6)}$. Essas proteínas exercem seus efeitos através de três receptores celulares: VEGFR-1, VEGFR-2 e VEGFR-3 ${ }^{(6)}$. Os VEGF-C e VEGF-D estão predominantemente envolvidos na linfangiogênese ${ }^{(7.8)}$. O VEGF-B atua no controle da degradação da matrix extra-celular e da adesão e migração celular ${ }^{(8)}$. O VEGFA é o principal fator encontrado na corrente sangüínea, e promove a formação de novos vasos por ações distintas e sinérgicas.

\section{VEGF-A}

Existem quatro isoformas conhecidas de VEGFA, produzidas pela fratura alternada do gene do VEGF, dando origem a proteínas com 121, 165, 189 e 208 aminoácidos ${ }^{(9)}$. O VEGF ${ }_{121}$ geralmente é encontrado na forma livre e parece ser essencial para os processos fisiológicos de manutenção da vascularização ocular ${ }^{(10)}$. O VEGF $_{165}$ é a principal isoforma encontrada no olho humano, e é considerado o maior responsável pela angiogênese em processos patológicos ${ }^{(9-1)}$. Encontra-se geralmente ligado à heparina, à superfície celular ou à matrix extra-celular ${ }^{(9)}$. Calcula-se que seus efeitos de estímulo à angiogênese são produzidos a partir da ligação aos receptores VEGFR-1 e VEGFR-2, ativando proteínas alvo intracelulares, principalmente a proteína kinase $\mathrm{C}^{(6-11)}$. Os $\mathrm{VEGF}_{189}$ e VEGF ${ }_{208}$ têm alta afinidade pela heparina e são encontrados sequestrados na matrix extra-celular ${ }^{(9)}$.

Extensas pesquisas em modelos animais de neovascularização da córnea, íris, retina e coróide concluíram que o VEGF é o mediador químico mais importante em diversas patologias oculares que cursam com neovascularização e aumento da permeabilidade vascular. Esses esforços permitiram estabelecer com clareza que os níveis de VEGF encontram-se elevados na neovascularização ocular ${ }^{(12-14)}$, que a elevação experimental dos níveis de VEGF induz a neovascularização ocular ${ }^{(15-1)}$ e que a inibição do VEGF pode impedir o desenvolvimento de neovasos ${ }^{(17-19)}$.

De fato, o VEGF-A participa do processo de angiogênese por vários mecanismos ${ }^{(20)}$ ( Tabela 1). Em primeiro lugar, promove um aumento do número de células endoteliais. Esse aumento é obtido a partir de dois caminhos distintos. Um destes é o estímulo direto à mitose de células endoteliais já existentes, que é mediado pela ligação ao receptor VEGFR-2 ${ }^{(21)}$. O outro baseia-se na atração de células precursoras da medula óssea e sua diferenciação em células endoteliais, o que depende da ligação ao receptor VEGFR-1 ${ }^{(2223)}$. Para que os neovasos sejam formados, é necessário que as novas 


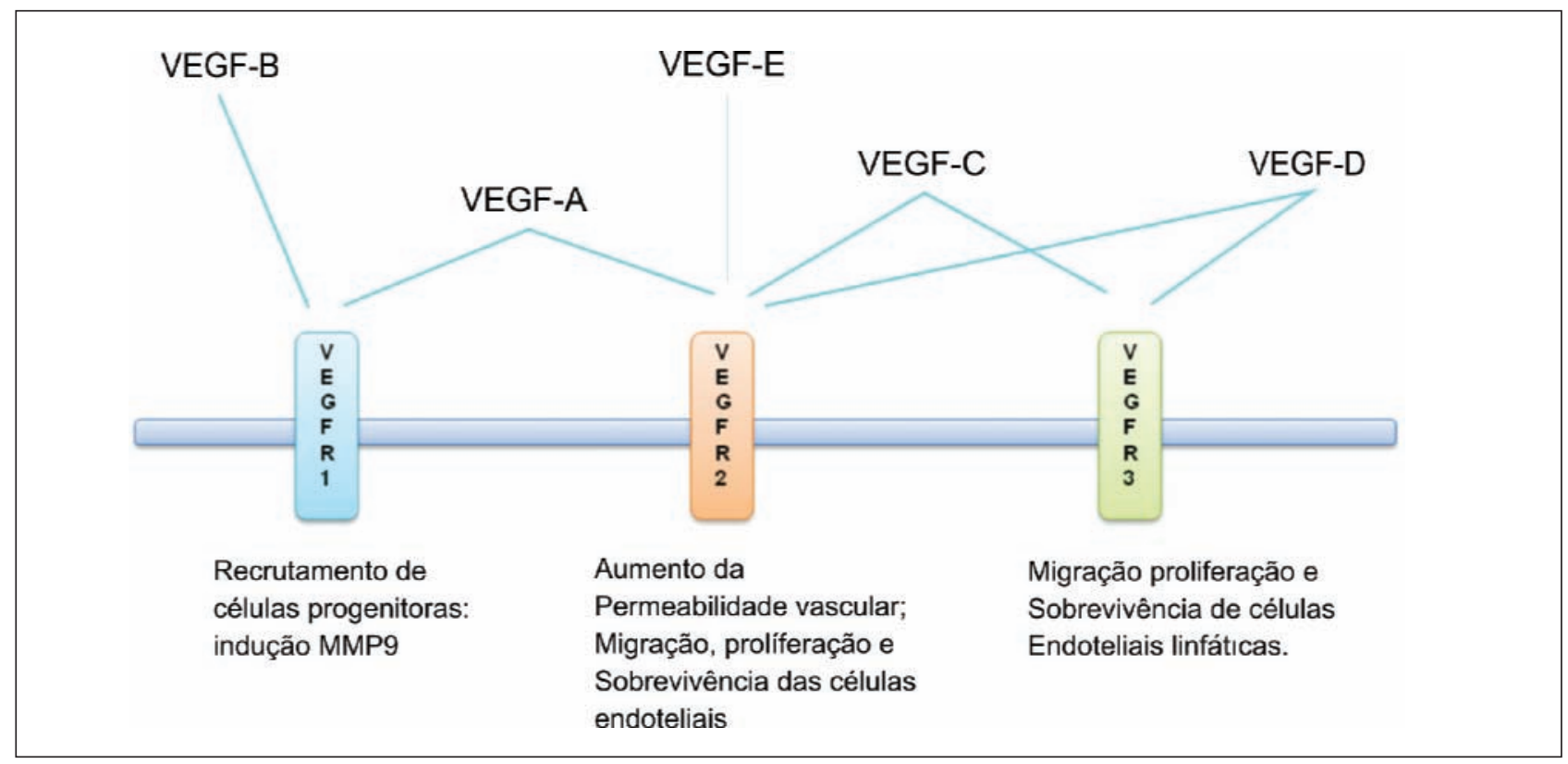

Figura 1: A família de fatores de crescimento derivados do endotélio (VEGF) e seus receptores (VEGFR) encontrados nas células endoteliais vasculares e linfáticas; MMP9 = matrix metaloproteinase ${ }^{(9)}$

células endoteliais consigam transpor o leito vascular e acessar o tecido isquêmico. O próprio VEGF aumenta a permeabilidade vascular pela formação de fenestrações endoteliais na microcirculação ${ }^{(24)}$. Finalmente, o VEGFA aumenta a expressão de metaloproteinases, enzimas proteolíticas que degradam a matriz extra-celular, abrindo espaço para a formação de neovasos ${ }^{(25)}$. Nesse processo, outras moléculas maiores de VEGF que se encontram sequestradas na matriz extra-celular $\left(\mathrm{VEGF}_{189} \mathrm{e}\right.$ $\mathrm{VEGF}_{208}$ ) são liberadas, potencializando ainda mais o estímulo pró-angiogênico ${ }^{(8)}$.

Além de promover a formação de novos vasos, existem evidências de que o VEGF-A também é importante para a manutenção destes, por preservar as células endoteliais recém-formadas, inibindo o mecanismo de apoptose nessas células ${ }^{(26)}$.Apesar de sua função principal ser o estímulo à angiogênese, o VEGF tem um espectro de ação bem diversificado, destacando-se também como agente pró-inflamatório. Este efeito se deve em parte ao aumento da permeabilidade vascular (fenestrações), mas também por promover a adesão leucocitária, através do estímulo à expressão de moléculas de adesão intercelulares 1 (ICAM-1) ${ }^{(27)}$.

É fundamental destacar que algumas isoformas do VEGF-A, particularmente o VEGF ${ }_{121}$, tem papel importante em processos fisiológicos. O VEGF tem propriedades neuroprotetoras já demonstradas, atribuídas em parte a sua capacidade de prolongar a sobrevida de células endoteliais, neurônios e células de Schwann ${ }^{(10-28)}$.
Por esse motivo, agentes que bloqueiam todas as isoformas de VEGF-A devem ser utilizados com cautela, uma vez que podem interferir em processos fisiológicos vitais e gerar um desequilíbrio que coloca em risco células e tecidos de extrema importância ${ }^{(20)}$.

\section{Níveis elevados de VEGF na neovascularização ocular}

Estudos já demonstraram que os níveis de VEGF encontram-se elevados nos fluídos oculares em doenças como retinopatia diabética proliferativa e na forma isquêmica da oclusão da veia central da retina ${ }^{(12-14,29)}$. Em um modelo experimental com primatas sobre neovascularização de íris secundária, a oclusão de veias retinianas induzida por laser e os níveis de VEGF eram diretamente proporcionais à intensidade da neovascularização ${ }^{(30)}$. Acredita-se que, no globo ocular, o epitélio pigmentário da retina é o principal regulador e produtor de VEGF em condições de hipóxia ${ }^{(31-32)}$.

\section{Elevação dos níveis de VEGF induz neovascularização ocular}

Estudos experimentais concluíram que um incremeto na expressão de VEGF é necessário e suficiente para promover neovascularização. Uma única injeção intravítrea de VEGF em olhos de primatas leva à neovascularização iriana, retiniana e pré-retiniana ${ }^{(15,16,}$ ${ }^{33)}$. Em outro estudo, observou-se que a razão $\mathrm{VEGF}_{164} /$ $\mathrm{VEGF}_{120}$ na retina de ratos com neovascularização retiniana induzida por isquemia era de $25.3 \pm 8.7$, en- 
quanto em condições fisiológicas normais, a razão $\mathrm{VEGF}_{164} / \mathrm{VEGF}_{120}$ não passa de $2.2 \pm 1.1^{(10)}$. Além disso, implantes de $\mathrm{VEGF}_{164}$ (forma equivalente a $\mathrm{VEGF}_{165}$ humana) em olhos de rato mostraram efeito pró-inflamatório, leucostático e de quebra da barreira endotelial mais potentes do que os de $\mathrm{VEGF}_{120}{ }^{(34)}$. Esses estudos permitiram um melhor entendimento da importância da isoforma $\mathrm{VEGF}_{165}$ na promoção da angiogênese em situações patológicas e seu efeito pró-inflamatório, colocando esta molécula como de alto interesse para controle destes processos.

\section{Inativação do VEGF impede a neovascularização ocular}

A participação do VEGF parece ser indispensável para a neovascularização ocular patológica. Diversos estudos estabeleceram claramente que o bloqueio do VEGF pode inibir a neovascularização da íris ${ }^{(17)}$, coróide ${ }^{(19)}$, córnea ${ }^{(35)}$ e retina ${ }^{(18-36)}$. Uma pesquisa experimental com primatas demonstrou que a injeção intravítrea de um anticorpo monoclonal antiVEGF previne a formação de neovasos de íris em um modelo de isquemia retiniana, após oclusão venosa induzida com laser ${ }^{(17)}$.

\section{Agentes AntiVEGF Bevacizumab}

O Bevacizumab (Avastin ; Genentech,Inc,San Francisco, Califórnia, USA) é um anticorpo monoclonal humanizado contra todas as isoformas de VEGF ${ }^{(37-38)}$. Esta substância foi desenvolvida e recebeu aprovação do FDA (fevereiro, 2004) para ser utilizada por via intravenosa no tratamento do câncer cólon-retal metastático ${ }^{\left({ }^{39}\right)}$. O Bevacizumab não é formalmente aprovado para uso intraocular e, até o momento, não existem resultados finais de grandes ensaios clínicos randomizados sobre essa forma de utilização. Por ter alto peso molecular (149 kD) acreditava-se que o Bevacizumab não atravessaria a interface vítreoretiniana para atuar nas neovascularizações corioretinianas. Entretanto, existem evidências clínicas crescentes de seu efeito positivo em diversas doenças oculares que cursam com neovascularização, como retinopatia diabética proliferativa ${ }^{(40)}$, oclusão vasculares da retina ${ }^{(40)}$ e degeneração macular relacionada à idade ${ }^{(22-43)}$,entre outras.

\section{Ranibizumab}

Ranibizumab (Lucentis $®$; Genentech, Inc, San Francisco, Califórnia, USA) é um fragmento de anticorpo monoclonal humanizado, com peso molecular de 48 $\mathrm{kDa}^{(4)}$. Assim como, o Bevacizumab atua bloqueando todas as isoformas de VEGF livres ${ }^{(4546)}$. Por ter menor peso
Tabela 1

\section{Propriedades do fator de crescimento derivado do endotélio (VEGF)}

\section{Propriedades do VEGF}

Estímulo à angiogênese;

Aumento da permeabilidade vascular, fenestração;

Efeito pró-inflamatório;

Efeito neuroprotetor;

Fator de sobrevivência vascular.

molecular, o Ranibizumab poderia atravessar com maior facilidade a interface vítreo-retiniana para atuar nas neovascularizações da retina e coróide, sendo esta sua principal vantagem sobre o Bevacizumab na utilização por via intravítrea.

\section{Pegaptanib}

Aprovado pelo FDA para o tratamento da DMRI em dezembro de 2004, o Pegaptanib (Macugen ${ }^{\circledR}$; Eyetech Pharmaceuticals, New York, New York, USA) faz parte de uma nova classe terapêutica: os aptâmeros ${ }^{(47)}$. Aptâmeros são oligonucleotídeos com uma conformação tridimensional específica que permite sua ligação a alvos moleculares com alta afinidade e especificidade. Ao contrário dos outros agentes antiVEGF,o Pegaptanib bloqueia apenas a isoforma $\mathrm{VEGF}_{165}$, considerada responsável pela neovascularização em processos patológicos ${ }^{(48)}$. Pelo menos, teoricamente, esta ação seletiva seria vantajosa, uma vez que outras isoformas de VEGF permaneceriam livres para participar de processos fisiológicos vitais.

\section{VEGF e antiVEGF no glaucoma}

Inúmeras publicações dão parte dos resultados positivos dos antiVEGF em patologias oculares que cursam com neovascularização. A maioria dessas pesquisas são voltadas para neovascularizações do segmento posterior, como a retinopatia diabética, as oclusões vasculares da retina e a DMRI. Embora, em menor escala, a utilização dos antiVEGF nas neovascularizações do segmento anterior, sobretudo no glaucoma neovascular, também tem sido objeto de interesse das pesquisas.

\section{AntiVEGF na neovascularização da íris}

A utilização de Bevacizumab por via intravítrea e intracameral já se mostrou eficaz em reduzir a neovascularização de íris, segundo algumas publicações. Em 2005, Avery ${ }^{(49)}$ relatou um caso de retinopatia 
diabética proliferativa com neovascularização de íris tratado com injeção intravítrea de 1,25 mg de Bevacizumab, no Congresso da Academia Americana de Oftalmologia. Após uma semana, tanto os neovasos da retina quanto os da íris haviam regredido e não eram mais visíveis à biomicroscopia ou à angiofluoresceinografia.

Oshima et al. ${ }^{(40)}$ observaram resultado semelhante em uma série de 7 casos de retinopatia diabética proliferativa com neovasos de íris tratados com Bevacizumab intravítreo e publicaram seus achados no American Journal of Ophthalmology em 2006. A regressão dos neovasos da íris ocorreu em todos os casos no intervalo de uma semana. Entretanto, após dois meses, dois olhos apresentaram recorrência. Uma segunda aplicação foi realizada com estabilização do quadro.

Davidorf et al. ${ }^{(50)}$ reportaram o caso de um paciente diabético com história de melanoma de coróide tratado com termoterapia transpupilar e radioterapia externa que evoluiu com glaucoma neovascular no olho contralateral. Após injeção intravítrea de $1,25 \mathrm{mg}$ de Bevacizumab observou-se regressão dos neovasos da íris e do ângulo. O paciente foi submetido à trabeculectomia 3 meses após a injeção intravítrea, com resultado surpreendentemente satisfatório diante dessas circunstâncias.

\section{AntiVEGF no glaucoma neovascular}

A concentração de VEGF encontra-se elevada no vítreo e no humor aquoso de pacientes com retinopatia diabética proliferativa.

Em 2006, Grisanti et al. ${ }^{(51)}$ utilizou $1.0 \mathrm{mg}$ de Bevacizumab intracameral em 6 olhos de 3 pacientes com glaucoma neovascular secundário a retinopatia diabética (2) e oclusão da veia central da retina (1). A angiografia fluoresceínica da íris revelou redução do extravasamento vascular no dia seguinte à administração de Bevacizumab. Nenhum efeito colateral foi observado durante o período de acompanhamento (4 semanas). A partir desses resultados, os autores passaram a sugerir a utilização de Bevacizumab intracameral como importante estratégia no tratamento da rubeose de íris e glaucoma neovascular.

Batioglu et al. ${ }^{(52)}$ reportaram um caso de glaucoma neovascular secundário à retinopatia diabética em que a panfotocoagulação retiniana não foi possível por visibilização inadequada do fundo. Uma semana após injeção intravítrea de Bevacizumab houve regressão dos neovasos, redução da pressão intra-ocular e melhora da transparência dos meios oculares, o que permitiu retomar a laserterapia. Concluíram que o Bevacizumab deve ser considerado como importante coadjuvante à panfotocoagulação retiniana no tratamento do glaucoma neovascular secundário à retinopatia diabética.

Chilov et al. ${ }^{(53)}$ relataram três casos de glaucoma neovascular tratados com Bevacizumab intravítreo que evoluíram com melhora da neovascularização e redução da pressão intra-ocular. Um dos olhos tratados apresentava hipoestesia corneana relacionada ao diabetes e desenvolveu úlcera de córnea após o tratamento, o que os autores atribuíram ao bloqueio da ação neurotrófica do VEGF pelo Bevacizumab.

Kahook et al. ${ }^{(54)}$ utilizaram $1,0 \mathrm{mg}$ de Bevacizumab intravítreo num caso de glaucoma neovascular secundário à oclusão da veia central da retina. A injeção foi indicada após insucesso no controle da pressão intra-ocular com terapia clínica, panfotocoagulação retiniana e ciclofotocoagulação transescleral. Observou-se redução da pressão intra-ocular e melhora do conforto a partir de dois dias após a injeção de Bevacizumab. Os autores não concluíram qual seria o mecanismo responsável pela redução da pressão intra-ocular neste caso.

De fato, a redução de pressão intra-ocular observada após aplicação intravítrea de Bevacizumab é motivo de especulação. Em olhos com ângulo parcialmente aberto, essa redução poderia ser explicada pela regressão dos neovasos, com melhora da filtração trabecular. Entretanto, em casos mais avançados, com formação de sinéquias anteriores extensas, essa hipótese isoladamente não explica os resultados observados ${ }^{(55)}$.

Iliev et al. ${ }^{(56)}$ descreveram uma série de 6 casos de glaucoma neovascular secundário à oclusão da veia central da retina com pressão intra-ocular muito elevada, sintomática e refratária ao tratamento clínico. Todos foram tratados com 1,25 mg de Bevacizumab intravítreo, o que resultou em regressão dos neovasos e melhora dos sintomas após $48 \mathrm{~h}$. Panfotocoagulação retiniana foi realizada em todos os casos assim que as condições permitiram. Quando o controle da pressão intra-ocular com medicação tópica após a injeção intravítrea não foi alcançada, o que ocorreu em 3 casos, os pacientes foram submetidos à ciclofotocoagulação transescleral. Os autores não observaram nenhum efeito colateral relacionado ao uso de Bevacizumab.

Vatavuk et al. ${ }^{(57)}$ aplicaram 1,25 mg de Bevacizumab intravítreo num caso de glaucoma neovascular secundário à oclusão da artéria central da retina. Os neovasos da íris e do ângulo regrediram em 48 h, com redução da PIO de 30 para $15 \mathrm{mmHg}$ e melhora significativa do desconforto sentido pelo paciente. Quatro semanas após a injeção, o paciente foi submetido à panfotocoagulação retiniana como tratamento definitivo. 


\section{Variação da PIO após Bevacizumab intravítreo}

Um dos motivos de preocupação quanto ao uso de Bevacizumab em pacientes glaucomatosos reside na questão da elevação da pressão intra-ocular imediatamente após a administração intravítrea. Hollands et al ${ }^{(58)}$ dirigiram um estudo para avaliar a flutuação da pressão intraocular nos minutos seguintes à aplicação intravítrea em 104 pacientes/104 olhos com indicação de Bevacizumab (tratamento de DMRI, retinopatia diabética, entre outros). Observaram que 2 minutos após a injeção de $0,05 \mathrm{ml}(1,25$ $\mathrm{mg}$ de Bevacizumab), 13 pacientes (12,5\%) apresentaram PIO acima de $50 \mathrm{mmHg}$, sendo que em 3 destes a PIO estava acima de $70 \mathrm{mmHg}$.Ao final de 30 minutos, quatorze pacientes mantinham pressão intra-ocular $5 \mathrm{mmHg}$ acima da medida inicial e 3 pacientes ainda apresentavam pressão intra-ocular acima de $25 \mathrm{mmHg}$. A análise regressiva com múltiplas variáveis mostrou uma tendência de pacientes fácicos apresentarem PIO mais elevadas ao final dos 30 minutos. Apenas 16 dos 104 pacientes apresentavam história prévia de glaucoma e curiosamente este fato não interferiu na variação da PIO após a injecção de acordo com a análise estatística. Os autores concluíram que a conseqüência destas flutuações súbitas e fugazes é desconhecida, e que um pequeno número de pacientes mantiveram pressão intra-ocular elevada (acima de $25 \mathrm{mmHg}$ ) por mais de 30 minutos, sugerindo como precaução avaliar a PIO de todos os pacientes após administração intravítrea de Bevacizumab. A elevação da pressão intra-ocular estaria relacionada ao aumento abrupto do conteúdo da cavidade vítrea sem tempo suficiente para a compensação pelos mecanismos fisiológicos de controle da PIO.

\section{AntiVEGF no tratamento cirúrgico do glaucoma}

A angiogênese é parte fundamental do processo de reparação tecidual ${ }^{(59)}$. A falha de controle do processo cicatricial no período pós-operatório é atualmente a maior limitação das cirurgias fistulizantes antiglaucomatosas. A mitomicina-C e o 5-fluorouracil se apresentaram como boas alternativas para evitar a fibrose pós-operatória ${ }^{(6-6)-61)}$, embora tenham vindo acompanhados de um grande número de complicações. Os antiVEGF também poderiam servir como coadjuvantes no controle da cicatrização pósoperatória em casos específicos.

Jonas et al ${ }^{(62)}$ relataram 2 casos de pacientes que foram submetidos à trabeculectomia convencional com injeção intravítrea de $1,5 \mathrm{mg}$ de Bevacizumab. Até a 12 semana de pós-operatório, a pressão intra-ocular se manteve entre 10 e $14 \mathrm{mmHg}$ nos dois casos, o que levou os autores a considerarem o Bevacizumab como coadjuvante na trabeculectomia.

Kahook et al. ${ }^{63}$ reportaram um caso de agulhamento em bolha encapsulada com 1,0 $\mathrm{mg}$ de Bevacizumab, após insucesso no agulhamento com mitomicina-C. Observou que, após o procedimento, a bolha se tornou mais difusa e menos vascularizada, sugerindo que esta pode ser uma boa indicação para o uso de Bevacizumab.

\section{Conclusão}

O controle da angiogênese é uma estratégia promissora no tratamento das neovascularizações do segmento anterior, tanto na fase de neovascularização da íris e do ângulo quanto no glaucoma neovascular. Entretanto, se a origem da isquemia não for resolvida, a produção local de VEGF continuará estimulando a angiogênese, podendo ocorrer o ressurgimento dos neovasos. Na maioria destas situações, os antiVEGF podem atuar como coadjuvantes importantes, criando condições que possibilitam um tratamento definitivo.

O controle da cicatrização pós-cirúrgica é uma nova aplicação destas substâncias, ainda pouco estudada. Relatos recentes sugerem que estas substâncias podem contribuir para o sucesso das cirurgias antiglaucomatosas, assim como resgatar casos de bolhas filtrantes em falência.

Vários pontos permanecem por serem esclarecidos, como a duração de ação, a toxicidade, a eficácia e a dose ideal de cada uma dessas substâncias. Estudos experimentais e ensaios clínicos randomizados e controlados dirigidos a estas questões são necessários para estabelecer qual é o verdadeiro papel e as possíveis aplicações dos antiVEGF nas patologias oculares.

\section{Abstract}

Angiogenesis is a complex process that results in new blood vessel formation. It is involved in physiological and pathological conditions and participates in several eye diseases. Among the chemical mediators of angiogenesis, the vascular endothelial growth factor $(V E G F)$ is recognized as one of the most important. In the last years, anti-VEGF agents have been introduced for the treatment of neovascularization of the posterior segment with very positive results. Some authors demonstrated benefits of using these substances for the control of glaucoma in specific situations.

Keywords: Angiogenesis inhibitors/therapeutic use; Neovascularization, pathologic/drug therapy; Glaucoma, neovascular/drug therapy; Vascular endothelial growth factors; Review 


\section{REFERÊNCIAS}

1. Folkman J. Tumor angiogenesis: therapeutic implications. N Engl J Med. 1971;285(21):1182-6.

2. Carmeliet P. Angiogenesis in health and disease. Nat Med. 2003;9(6):653-60.

3. Cleaver O, Melton DA. Endothelial signaling during development. Nat Med. 2003;9(6):661-8.

4. Levy AP, Levy NS, Wegner S, Goldberg MA. Transcriptional regulation of the rat vascular endothelial growth factor gene by hypoxia. J Biol Chem. 1995;270(22):13333-40.

5. Thornton AD, Ravn P, Winslet M, Chester K. Angiogenesis inhibition with Bevacizumab and the surgical management of colorectal cancer. Br J Surg. 2006;93(12):1456-63.

6. Ferrara N, Gerber HP, LeCouter J. The biology of VEGF and its receptors. Nat Med. 2003;9(6):669-76.

7. Takahashi M, Yoshimoto T, Kubo H. Molecular mechanisms of lymphangiogenesis. Int J Hematol. 2004;80(1):29-34

8. Veikkola T, Alitalo K. VEGFs, receptors and angiogenesis. Semin Cancer Biol. 1999;9(3):211-20.

9. Park JE, Keller GA, Ferrara N. The vascular endothelial growth factor (VEGF) isoforms: differential deposition into the subepithelial extracellular matrix and bioactivity of extracellular matrix-bound VEGF. Mol Biol Cell. 1993;4(12):1317-26.

10. Ishida S, Usui T, Yamashiro K, Kaji Y, Amano S, Ogura Y, et al. VEGF164-mediated inflammation is required for pathological, but not physiological, ischemia-induced retinal neovascularization. J Exp Med. 2003;198(3):483-9.

11. Ferrara N. Vascular endothelial growth factor: basic science and clinical progress. Endocr Rev. 2004;25(4):581-611.

12. Adamis AP, Miller JW, Bernal MT, D’Amico DJ, Folkman J, Yeo TK, et al. Increased vascular endothelial growth factor levels in the vitreous of eyes with proliferative diabetic retinopathy. Am J Ophthalmol. 1994;118(4):445-50.

13. Aiello LP, Avery RL, Arrigg PG, Keyt BA, Jampel HD, Shah ST, et al. Vascular endothelial growth factor in ocular fluid of patients with diabetic retinopathy and other retinal disorders. N Engl J Med. 1994;331(22):1480-7.

14. Brooks HL Jr, Caballero S Jr, Newell CK, Steinmetz RL, Watson D, Segal MS, et al. Vitreous levels of vascular endothelial growth factor and stromal-derived factor 1 in patients with diabetic retinopathy and cystoid macular edema before and after intraocular injection of triamcinolone. Arch Ophthalmol. 2004 Dec;122(12):1801-7.

15. Tolentino MJ, Miller JW, Gragoudas ES, Jakobiec FA, Flynn $\mathrm{E}$, Chatzistefanou $\mathrm{K}$, et al. Intravitreous injections of vascular endothelial growth factor produce retinal ischemia and microangiopathy in an adult primate. Ophthalmology. 1996;103(11):1820-8.

16. Tolentino MJ, Miller JW, Gragoudas ES, Chatzistefanou K, Ferrara N, Adamis AP. Vascular endothelial growth factor is sufficient to produce iris neovascularization and neovascular glaucoma in a nonhuman primate. Arch Ophthalmol. 1996;114(8):964-70.

17. Adamis AP, Shima DT, Tolentino MJ, Gragoudas ES, Ferrara $\mathrm{N}$, Folkman J, et al. Inhibition of vascular endothelial growth factor prevents retinal ischemia-associated iris neovascularization in a nonhuman primate. Arch Ophthalmol. 1996;114(1):66-71.

18. Aiello LP, Pierce EA, Foley ED, Takagi H, Chen H, Riddle L, et al. Suppression of retinal neovascularization in vivo by inhibition of vascular endothelial growth factor (VEGF) using soluble VEGF-receptor chimeric proteins. Proc Natl Acad Sci U S A. 1995;92(23):10457-61.
19. Krzystolik MG, Afshari MA, Adamis AP, Gaudreault J, Gragoudas ES, Michaud NA, et al. Prevention of experimental choroidal neovascularization with intravitreal anti-vascular endothelial growth factor antibody fragment. Arch Ophthalmol. 2002;120(3):338-46.

20. Ng EW, Adamis AP. Targeting angiogenesis, the underlying disorder in neovascular age-related macular degeneration. Can J Ophthalmol. 2005;40(3):352-68.

21. Leung DW, Cachianes G, Kuang WJ, Goeddel DV, Ferrara N. Vascular endothelial growth factor is a secreted angiogenic mitogen. Science. 1989;246(4935):1306-9.

22. Hattori K, Heissig B, Wu Y, Dias S, Tejada R, Ferris B, et al. Placental growth factor reconstitutes hematopoiesis by recruiting VEGFR1(+) stem cells from bone-marrow microenvironment. Nat Med. 2002;8(8):841-9.

23. Asahara T, Takahashi T, Masuda H, Kalka C, Chen D, Iwaguro $\mathrm{H}$, et al. VEGF contributes to postnatal neovascularization by mobilizing bone marrow-derived endothelial progenitor cells. EMBO J. 1999;18(14):3964-72.

24. Roberts WG, Palade GE. Increased microvascular permeability and endothelial fenestration induced by vascular endothelial growth factor. J Cell Sci. 1995;108 ( Pt 6):2369-79.

25. Hiratsuka S, Nakamura K, Iwai S, Murakami M, Itoh T, Kijima $\mathrm{H}$, et al. MMP9 induction by vascular endothelial growth factor receptor-1 is involved in lung-specific metastasis. Cancer Cell. 2002;2(4):289-300.

26. Alon T, Hemo I, Itin A, Pe'er J, Stone J, Keshet E. Vascular endothelial growth factor acts as a survival factor for newly formed retinal vessels and has implications for retinopathy of prematurity. Nat Med. 1995;1(10):1024-8.

27. Ishida S, Usui T, Yamashiro K, Kaji Y, Ahmed E, Carrasquillo $\mathrm{KG}$, et al. VEGF164 is proinflammatory in the diabetic retina. Invest Ophthalmol Vis Sci. 2003;44(5):2155-62.

28. Sondell M, Lundborg G, Kanje M. Vascular endothelial growth factor has neurotrophic activity and stimulates axonal outgrowth, enhancing cell survival and Schwann cell proliferation in the peripheral nervous system. J Neurosci. 1999;19(14):5731-40.

29. Sawada O, Kawamura H, Kakinoki M, Sawada T, Ohji M. Vascular endothelial growth factor in aqueous humor before and after intravitreal injection of Bevacizumab in eyes with diabetic retinopathy. Arch Ophthalmol. 2007;125(10):1363-6.

30. Miller JW, Adamis AP, Shima DT, D'Amore PA, Moulton RS, O'Reilly MS, et al. Vascular endothelial growth factor/ vascular permeability factor is temporally and spatially correlated with ocular angiogenesis in a primate model. Am J Pathol. 1994;145(3):574-84.

31. Blaauwgeers HG, Holtkamp GM, Rutten H, Witmer AN, Koolwijk P, Partanen TA, et al. Polarized vascular endothelial growth factor secretion by human retinal pigment epithelium and localization of vascular endothelial growth factor receptors on the inner choriocapillaris. Evidence for a trophic paracrine relation. Am J Pathol. 1999;155(2):421-8.

32. Aiello LP, Northrup JM, Keyt BA, Takagi H, Iwamoto MA. Hypoxic regulation of vascular endothelial growth factor in retinal cells. Arch Ophthalmol. 1995;113(12):1538-44.

33. Tolentino MJ, McLeod DS, Taomoto M, Otsuji T, Adamis AP, Lutty GA. Pathologic features of vascular endothelial growth factor-induced retinopathy in the nonhuman primate. Am J Ophthalmol. 2002;133(3):373-85.

34. Usui T, Ishida S, Yamashiro K, Kaji Y, Poulaki V, Moore J, et al. VEGF164(165) as the pathological isoform: differential leukocyte and endothelial responses through VEGFR1 and VEGFR2. Invest Ophthalmol Vis Sci. 2004;45(2):368-74. 
35. Amano S, Rohan R, Kuroki M, Tolentino M, Adamis AP. Requirement for vascular endothelial growth factor in woundand inflammation-related corneal neovascularization. Invest Ophthalmol Vis Sci. 1998;39(1):18-22.

36. Honda M, Sakamoto T, Ishibashi T, Inomata H, Ueno H. Experimental subretinal neovascularization is inhibited by adenovirus-mediated soluble VEGF/flt-1 receptor gene transfection: a role of VEGF and possible treatment for SRN in age-related macular degeneration. Gene Ther. 2000;7(11):978-85.

37. Ferrara N, Hillan KJ, Gerber HP, Novotny W. Discovery and development of Bevacizumab, an anti-VEGF antibody for treating cancer. Nat Rev Drug Discov. 2004;3(5):391-400.

38. Ferrara N, Hillan KJ, Novotny W. Bevacizumab (Avastin), a humanized anti-VEGF monoclonal antibody for cancer therapy. Biochem Biophys Res Commun. 2005;333(2):328-35.

39. Hurwitz H, Fehrenbacher L, Novotny W, Cartwright T, Hainsworth J, Heim W, et al. Bevacizumab plus irinotecan, fluorouracil, and leucovorin for metastatic colorectal cancer. N Engl J Med. 2004;350(23):2335-42.

40. Oshima Y, Sakaguchi H, Gomi F, Tano Y. Regression of iris neovascularization after intravitreal injection of Bevacizumab in patients with proliferative diabetic retinopathy. Am J Ophthalmol. 2006;142(1):155-8.

41. Rosenfeld PJ, Fung AE, Puliafito CA. Optical coherence tomography findings after an intravitreal injection of Bevacizumab (avastin) for macular edema from central retinal vein occlusion. Ophthalmic Surg Lasers Imaging. 2005;36(4):336-9.

42. Rosenfeld PJ, Moshfeghi AA, Puliafito CA. Optical coherence tomography findings after an intravitreal injection of Bevacizumab (avastin) for neovascular age-related macular degeneration. Ophthalmic Surg Lasers Imaging. 2005;36(4):331-5.

43. Avery RL, Pieramici DJ, Rabena MD, Castellarin AA, Nasir MA, Giust MJ. Intravitreal Bevacizumab (Avastin) for neovascular age-related macular degeneration. Ophthalmology. 2006;113(3):363-372.e5.

44. Ferrara N, Damico L, Shams N, Lowman H, Kim R Development of ranibizumab, an anti-vascular endothelial growth factor antigen binding fragment, as therapy for neovascular age-related macular degeneration. Retina. 2006;26(8):859-70.

45. Heier JS, Antoszyk AN, Pavan PR, Leff SR, Rosenfeld PJ, Ciulla TA, et al. Ranibizumab for treatment of neovascular age-related macular degeneration: a phase I/II multicenter, controlled, multidose study. Ophthalmology. 2006 Apr;113(4):633.e1-4.

46. Rosenfeld PJ, Brown DM, Heier JS, Boyer DS, Kaiser PK, Chung CY, et al. Ranibizumab for neovascular age-related macular degeneration. N Engl J Med. 2006;355(14):1419-31.

47. Ruckman J, Green LS, Beeson J, Waugh S, Gillette WL, Henninger DD, et al. 2'-Fluoropyrimidine RNA-based aptamers to the 165-amino acid form of vascular endothelial growth factor (VEGF165). Inhibition of receptor binding and VEGF-induced vascular permeability through interactions requiring the exon 7 -encoded domain. J Biol Chem. 1998;273(32):20556-67.

48. Gragoudas ES, Adamis AP, Cunningham ET Jr, Feinsod M, Guyer DR; VEGF Inhibition Study in Ocular Neovascularization Clinical Trial Group. Pegaptanib for neovascular age-related macular degeneration. N Engl J Med. 2004;351(27):2805-16.
49. Avery RL. Regression of retinal and iris neovascularization after intravitreal Bevacizumab (Avastin) treatment. Retina. 2006;26(3):352-4.

50. Davidorf FH, Mouser JG, Derick RJ. Rapid improvement of rubeosis iridis from a single Bevacizumab (Avastin) injection. Retina. 2006;26(3):354-6.

51. Grisanti S, Biester S, Peters S, Tatar O, Ziemssen F, BartzSchmidt KU et al. Intracameral Bevacizumab for iris rubeosis. Am J Ophthalmol. 2006;142(1):158-60.

52. Batioðlu F, Astam N, Ozmert E. Rapid improvement of retinal and iris neovascularization after a single intravitreal Bevacizumab injection in a patient with central retinal vein occlusion and neovascular glaucoma. Int Ophthalmol. 2008;28(1):59-61.

53. Chilov MN, Grigg JR, Playfair TJ. Bevacizumab (Avastin) for the treatment of neovascular glaucoma. Clin Experiment Ophthalmol. 2007;35(5):494-6.

54. Kahook MY, Schuman JS, Noecker RJ. Intravitreal Bevacizumab in a patient with neovascular glaucoma. Ophthalmic Surg Lasers Imaging. 2006;37(2):144-6.

55. Yazdani S, Hendi K, Pakravan M. Intravitreal Bevacizumab (Avastin) injection for neovascular glaucoma. J Glaucoma. 2007;16(5):437-9.

56. Iliev ME, Domig D, Wolf-Schnurrbursch U, Wolf S, Sarra GM. Intravitreal Bevacizumab (Avastin) in the treatment of neovascular glaucoma. Am J Ophthalmol. 2006;142(6):1054-6.

57. Vatavuk Z, Bencic G, Mandic Z. Intravitreal Bevacizumab for neovascular glaucoma following central retinal artery occlusion. Eur J Ophthalmol. 2007;17(2):269-71.

58. Hollands H, Wong J, Bruen R, Campbell RJ, Sharma S, Gale J. Short-term intraocular pressure changes after intravitreal injection of Bevacizumab. Can J Ophthalmol. 2007;42(6):807-11.

59. Ferrara N, Keyt B. Vascular endothelial growth factor: basic biology and clinical implications. EXS. 1997;79:209-32.

60. Chen CW, Huang HT, Bair JS, Lee CC. Trabeculectomy with simultaneous topical application of mitomycin-C in refractory glaucoma. J Ocul Pharmacol. 1990;6(3):175-82.

61. Singh K, Mehta K, Shaikh NM, Tsai JC, Moster MR, Budenz DL, et al. Trabeculectomy with intraoperative mitomycin C versus 5-fluorouracil. Prospective randomized clinical trial. Ophthalmology. 2000;107(12):2305-9.

62. Jonas JB, Spandau UH, Schlichtenbrede F. Intravitreal Bevacizumab for filtering surgery. Ophthalmic Res. 2007;39(2):121-2.

63. Kahook MY, Schuman JS, Noecker RJ. Needle bleb revision of encapsulated filtering bleb with Bevacizumab. Ophthalmic Surg Lasers Imaging. 2006;37(2):148-50. 